\title{
A fully temperature controlled test chamber for the application of gas sensor characterization
}

\begin{abstract}
Research and development on gas sensors design and fabrication demands the needs for test chambers as the characterizing and testing of gas sensor are based on its detection of the concentration of different type of gas under the influence of temperature and also humidity. This project, a Fully Temperature Controlled Test Chamber is about the design and development of a system to provide an artificial environment for gas sensor characterization. The main part of the designed system is the temperature feed back loop control system. Its function is to monitor and regulate the environment temperature to the desired value for characterization of gas sensor under test. While the LM35DZ temperature sensor is used for this purpose, the intended chamber system to be designed is able to communicate with control switches from outside of the chambers using keypad and LCD. The user can set the desired temperature in the chamber, and then the PIC16F877A microcontroller which acts the "brain" of this system will analyze and process the input signal from the sensor and keypad to give the corresponding output to control the heater and display on the LCD screen. When the steady state condition has been reached, the chamber will be ready for the testing of gas sensors under test. The inlet valve, vacuum pump and fan integrated in the chamber are also fully controlled by the microcontroller. Beside, the control system can also be controlled manually by using the manual switches. When tested using the sensor under test, the test chamber and the regulation system of the temperature are working successfully as programmed and give the desired outputs.
\end{abstract}

Keyword: Gas sensor characterization; Test chambers; Gas sensors; Fully Temperature Controlled Test Chamber; Temperature 